\title{
CHARACTERIZATIONS OF URYSOHN-CLOSED SPACES
}

\author{
LARRY L. HERRINGTON
}

\begin{abstract}
This paper gives characterizations of Urysohn-closed and
\end{abstract} minimal Urysohn spaces, some of which make use of nets.

1. Introduction. Our primary interest is the investigation of Urysohn-closed and minimal Urysohn spaces. Characterizations of Urysohn-closed and minimal Urysohn spaces are given in terms of special types of open filterbases [1, p. 101]. Open filterbases, of course, determine nets but not every net determines an open filterbase. We give characterizations of Urysohn-closed and minimal Urysohn spaces in terms of nets and arbitrary filterbases. These characterizations are obtained mainly through the introduction of a type of convergence for filterbases and nets that we call $u$-convergence.

Throughout, $\operatorname{cl}(A)$ will denote the closure of a set $A$.

2. Preliminary definitions and theorems. Let $X$ be a topological space and let $G$ and $H$ be open sets in $X$ containing a point $p \in X$. Then $G$ and $H$ will be called an ordered pair of open sets containing $p$ (denoted by $(G, H)$ ) if $p \in G \subset \operatorname{cl}(G) \subset H$.

Definition 2.1. Let $X$ be a topological space and let $\mathscr{F}=\left\{A_{\alpha}: \alpha \in \Delta\right\}$ be a filterbase in $X$. Then $\mathscr{F} u$-converges to $x \in X\left(\mathscr{F} \rightarrow_{u} x\right)$ if for each ordered pair of open sets $(G, H)$ containing $x$ there exists an $A_{\alpha} \in \mathscr{F}$ such that $A_{\alpha} \subset \operatorname{cl}(H)$. The filterbase $\mathscr{F} u$-accumulates to $x \in X\left(\mathscr{F} \propto_{u} x\right)$ if for each ordered pair of open sets $(G, H)$ containing $x$ and for each $A_{\alpha} \in \mathscr{F}$, $A_{\alpha} \cap \operatorname{cl}(H) \neq \varnothing$.

Convergence and accumulation of filterbases in the usual sense, of course, imply $u$-convergence and $u$-accumulation, respectively. However, the converses do not hold as the next example shows.

EXAMPLE 2.2. Let $I=[0,1]$ have as a subbase the usual open sets together with the set $A=\{r: 1 / 4<r<3 / 4$ and $r$ is rational $\}$. Let the filterbase $\mathscr{F}$ consist of a single element $B=\{x: 1 / 3<x<2 / 3$ and $x$ is irrational $\}$ and let $x=1 / 2$. The filterbase $\mathscr{F}$ does not converge or accumulate in the usual sense to $x$ but $\mathscr{F} \propto_{u} x$.

There are a number of theorems concerning $u$-convergence and $u$ accumulation whose statements parallel those of convergence and accumulation in the usual sense. We give a sample of some of these theorems but omit their straightforward proofs.

Received by the editors October 28, 1974.

AMS (MOS) subject classifications (1970). Primary 54D30.

Key words and phrases. Urysohn-closed and minimal Urysohn spaces.

○ American Mathematical Society 1976 
THEOREM 2.3. In a topological space $X$ the following properties hold:

(a) If $\mathscr{F}$ is a filterbase in $X$ such that $\mathscr{F} u$-converges to $x \in X$, then $\mathscr{F}$ $u$-accumulates to $x$. If $X$ is a Urysohn space and if $\mathscr{F}$ converges to $x \in X$, then $\mathscr{F} u$-accumulates at no point other than $x$.

(b) Let $\mathscr{F}_{1}$ and $\mathscr{F}_{2}$ be two filterbases in $X$ where $\mathscr{F}_{2}$ is stronger than $\mathscr{F}_{1}$. Then $\mathscr{F}_{1} u$-accumulates to $x \in X$ if $\mathscr{F}_{2} u$-accumulates to $x$.

(c) A filterbase $\mathscr{F}_{1} u$-accumulates to $x \in X$ if and only if there exists a filterbase $\mathscr{F}_{2}$ stronger than $\mathscr{F}_{1}$ such that $\mathscr{F}_{2} u$-converges to $x$.

(d) A maximal filterbase $\Re$ in $X u$-accumulates to $x \in X$ if and only if $\Re$ $u$-converges to $x$.

Definition 2.4. Let $X$ be a topological space and let $\theta: D \rightarrow X$ be a net in $X$. Then $\theta u$-converges to $x \in X\left(\theta \rightarrow_{u} x\right)$ if for each ordered pair of open sets $(G, H)$ containing $x$, there exists a $b \in D$ such that $\theta\left(T_{b}\right) \subset \operatorname{cl}(H)$ (where $\left.T_{b}=\{c \in D: b \prec c\}\right)$. The net $\theta u$-accumulates to $x \in X\left(\theta \propto_{u} x\right)$ if for each ordered pair of open sets $(G, H)$ containing $x$ and for every $b \in D, \theta\left(T_{b}\right) \cap \operatorname{cl}(H) \neq \varnothing$.

Of course, if $\vartheta: D \rightarrow X$ is a net in $X$, the family $\mathscr{F}(\vartheta)=\left\{\vartheta\left(T_{b}\right): b \in D\right\}$ is a filterbase in $X$ and it is routine to verify that:

(a) $\mathscr{F}(\theta) \rightarrow_{u} x \in X$ if and only if $\theta \rightarrow_{u} x$.

(b) $\mathscr{F}(\theta) \propto_{u} x \in X$ if and only if $\theta \propto_{u} x$.

Conversely, every filterbase $\mathscr{F}$ in $X$ determines a net $\theta: D \rightarrow X$ such that:

(a) $\mathscr{F} \rightarrow_{u} x \in X$ if and only if $\theta \rightarrow_{u} x$.

(b) $\mathscr{F} \propto_{u} x \in X$ if and only if $\theta \propto_{u} x$.

The construction of such a net is the same as that of [2, p. 213].

We next state a few theorems concerning $u$-convergence for nets.

THEOREM 2.5. In a topological space $X$ the following properties hold:

(a) If $\theta$ is a net in $X$ such that $\theta$ u-converges to $x \in X$, then $\theta$ $u$-accumulates to $x$. If $X$ is a Urysohn space and if $\mathcal{O}$ converges to $x \in X$, then $(0$ u-accumulates at no point other than $x$.

(b) A net $\theta$ u-accumulates to $x \in X$ if and only if there exists a subnet of $\theta$ $u$-converging to $x$.

(c) A universal net $\theta$ u-accumulates to $x \in X$ if and only if $\theta$ u-converges to $x$.

3. Filterbases and net characterizations of Urhysohn-closed spaces. An open filterbase base $\mathscr{F}$ in $X$ is a Urysohn filterbase if and only if for each $p \notin A(\mathscr{F})$ (where $A(\mathscr{F})$ denotes the set of accumulation points of $\mathscr{F}$ ), there is an open neighborhood $U$ of $p$ and some $V \in \mathscr{F}$ such that $\operatorname{cl}(U) \cap \operatorname{cl}(V)=\varnothing$ [3]. An open cover $\mathscr{Q}$ of a space $X$ is a Urysohn open cover if there exists an open cover $\mathcal{T}$ of $X$ with the property that for each $V \in \mathfrak{V}$, there is a $U \in$ Q such that $\operatorname{cl}(V) \subset U$. A Urysohn space $X$ is Urysohn-closed provided $X$ is a closed set in every Urysohn space in which it can be embedded [1].

Lemma 3.1. Let $\mathscr{F}=\left\{O_{\alpha}: \alpha \in \Delta\right\}$ be an open Urysohn filterbase on $X$. Then $A(\mathscr{F})=A_{u}(\mathscr{F})$ (where $A_{u}(\mathscr{F})$ denotes the set of $u$-accumulation points of $\left.\mathscr{F}\right)$.

Proof. Clearly we only need to show that $A_{u}(\mathscr{F}) \subset A(\widetilde{\mathscr{F}})$. Suppose $p \notin$ $A(\mathscr{F})$. Then there exists an open set $U$ containing $p$ and some $O_{\alpha} \in \mathscr{F}$ such 
that $\operatorname{cl}(U) \cap \operatorname{cl}\left(O_{\alpha}\right)=\varnothing$. The open sets $V=X-\operatorname{cl}\left(O_{\alpha}\right)$ and $U$ form an ordered pair of open sets, $(U, V)$, containing $p$ and have the property that $O_{\alpha} \cap \operatorname{cl}(V)=\varnothing$. Consequently, $p \notin A_{u}(\mathscr{F})$. Therefore, we conclude that $A(\mathscr{F})=A_{u}(\mathscr{F})$.

Theorem 4.1 of $[1$, p. 101] gives several characterizations of Urysohn-closed spaces. We offer the following characterizations.

THEOREM 3.2. Let $X$ be a Urysohn space. Then the following are equivalent:

(a) $X$ is Urysohn-closed.

(b) Each filterbase $\mathcal{F}$ in $X u$-accumulates to some point $x \in X$.

(c) Each maximal filterbase $\mathfrak{N}$ in $X$ u-converges to some point $x \in X$.

Proof. (a) implies (b). Suppose there exists a filterbase $\mathscr{F}=\left\{A_{\alpha} \in \Delta\right\}$ in $X$ that does not $u$-accumulate in $X$. Then for each $x \in X$ there exists an ordered pair of open sets $(U(x), V(x))$ containing $x$ and some $A_{\alpha(x)} \in \mathscr{F}$ such that $A_{\alpha(x)} \cap \operatorname{cl}(V(x))=\varnothing$. Now $\{V(x): x \in X\}$ is a Urysohn open cover of $X$. Thus by Theorem 4.1 of $\left[1\right.$, p. 101], there exists a finite subcollection $\left\{V\left(x_{i}\right)\right.$ : $i=1,2,3, \ldots, n\}$ such that $\cup_{i=1}^{n} \operatorname{cl}\left(V\left(x_{i}\right)\right)=X$. Since $\mathscr{F}$ is a filterbase, there exists an $A_{\alpha_{0}} \in \mathcal{F}$ such that $A_{\alpha_{0}} \subset \cap_{i=1}^{n} A_{\alpha\left(x_{i}\right)}$, and $A_{\alpha_{0}} \neq \varnothing$ implies that for some $j, 1 \leqslant j \leqslant n, A_{\alpha_{0}} \cap \operatorname{cl}\left(V\left(x_{j}\right)\right) \neq \varnothing$. Therefore $A_{\alpha\left(x_{j}\right)} \cap \operatorname{cl}\left(V\left(x_{j}\right)\right)$ $\neq \varnothing$ which is a contradiction.

(b) implies (a). Let $\mathscr{F}=\left\{O_{\alpha}: \alpha \in \Delta\right\}$ be an open Urysohn filterbase on $X$. By Lemma 3.1 and hypothesis (b) we have that $A_{u}(\mathscr{F})=A(\mathscr{F}) \neq \varnothing$. Therefore $X$ is Urysohn-closed according to Theorem 4.1 of [1, p. 101].

(b) implies (c). Let $\Re$ be a maximal filterbase in $X$. Then $\Re u$ accumulates to some point in $X$ by (b) and hence $u$-converges to that point by Theorem 2.3(d).

(c) implies (b). Let $\mathscr{F}$ be a filterbase in $X$. Then there exists a maximal filterbase $\mathfrak{N}$ in $X$ which is stronger than $\mathscr{F}$. Since $\mathfrak{R} u$-converges to some point $x \in X, \mathscr{F} u$-accumulates to $x$ according to Theorem 2.3.

Our discussion in the previous section showed that filterbases and nets are "equivalent" in the sense of $u$-convergence and $u$-accumulation. Thus we can now characterize Urysohn-closed spaces in terms of nets.

THEOREM 3.3. In a Urysohn space $X$ the following are equivalent:

(a) $X$ is Urysohn-closed.

(b) Each net in $X$ has a $u$-accumulation point.

(c) Each universal net u-converges.

REMARK 3.4. For each topological space $(X, \tau)$ there is a corresponding topological space $\left(X, \tau_{*}\right)$ called the semiregular space associated with $(X, \tau)$ $\left[1\right.$, p. 96]. The topology $\tau_{*}$ is generated by the regular open sets in $(X, \tau)$. For each open set $U$ in $(X, \tau), \operatorname{cl}(U)=\operatorname{cl}_{*}(U)$ (where $\operatorname{cl}_{*}(U)$ denotes the closure of $U$ in $\left.\left(X, \tau_{*}\right)\right)$. Consequently, it follows that a space $(X, \tau)$ is Urysohn if and only if $\left(X, \tau_{*}\right)$ is Urysohn. Also, it is easy to see that a filterbase $\mathscr{F}$ on $X$ $u$-accumulates to $x$ in $(X, \tau)$ if and only if $\widetilde{y} u$-accumulates to $x$ in $\left(X, \tau_{*}\right)$. With this in consideration we give the following theorem.

TheOREM 3.5. A space $(X, \tau)$ is Urysohn-closed if and only if $\left(X, \tau_{*}\right)$ is Urysohn-closed.

Proof. The result follows from Theorem 3.2 and Remark 3.4. 
Theorem 4.2 of $[1$, p. 101] characterizes minimal Urysohn spaces in terms of open Urysohn filterbases. In terms of arbitrary filterbases and $u$ convergence, we give the following characterization of minimal Urysohn spaces.

THEOREM 3.6. Let $\left(X, \tau_{0}\right)$ be a Urysohn space. Then $X$ is minimal Urysohn if and only if each filterbase in $X$ possessing at most one $u$-accumulation point is convergent.

Proof. Suppose the condition is given and let $\mathscr{F}$ be an open Urysohn filterbase on $X$ possessing at most one accumulation point. By Lemma 3.1, $\mathscr{F}$ possesses at most one $u$-accumulation point. Consequently, by hypothesis, $\mathscr{F}$ converges. This shows that $X$ is minimal Urysohn according to Theorem 4.2 of [1, p. 101].

Conversely, assume that $X$ is a minimal Urysohn space and suppose that $\mathscr{F}_{0}=\left\{A_{\alpha}: \alpha \in \Delta\right\}$ is a filterbase on $X$ possessing at most one $u$-accumulation point. Let $\mathcal{F}$ be the filter generated by the filterbase $\mathscr{F}_{0}$. Since $X$ is Urysohnclosed (see Theorem 4.3(a) of [1, p. 101]), $\mathscr{F}_{0}$ has a unique $u$-accumulation point $x \in X$. It follows that the collection of open sets $\tau_{1}=\left\{U \in \tau_{0}\right.$ : $U \subset X-\{x\}\} \cup\left\{V \in \tau_{0}: V \in \mathscr{F}\right\}$ forms a Urysohn topology on $X$ with the property that $\tau_{1} \subset \tau_{0}$. Suppose there is an open set $G(x) \in \tau_{0}$ containing $x$ such that for each $A_{\alpha} \in \mathscr{F}_{0}, A_{\alpha} \not \subset G(x)$. Then for each open $U(x) \in \tau_{1}$ containing $x, U(x) \not G(x)$ which shows that $\tau_{1} \neq \tau_{0}$. Therefore $\left(X, \tau_{0}\right)$ is not minimal Urysohn, which is a contradiction. We conclude that $\mathscr{F}_{0}$ converges to $x$.

Corollary 3.7. Let $X$ be a Urysohn space. Then $X$ is minimal Urysohn if and only if each net in $X$ possessing at most one u-accumulation point is convergent.

4. First countable Urysohn spaces. A space $(X, \tau)$ is called first countable and minimal Urysohn if $\tau$ is first countable and Urysohn, and if no first countable topology on $X$ which is strictly weaker than $\tau$ is Urysohn. $(X, \tau)$ is first countable and Urysohn-closed if $\tau$ is first countable and Urysohn, and $(X, \tau)$ is a closed subspace of every first countable Urysohn space in which it can be embedded.

Theorem 4.1. A first countable Urysohn space $X$ is first countable and Urysohn-closed if each countable filterbase on $X$ u-accumulates to some point $p \in X$.

Proof. Let $\mathcal{F}$ be a countable open Urysohn filterbase on $X$. By Lemma $3.1, A(\mathscr{F})=A_{u}(\mathscr{F}) \neq \varnothing$ which implies that $X$ is first countable and Urysohn-closed according to Theorem 6.3 of $[1$, p. 107].

THEOREM 4.2. A first countable Urysohn space $X$ is first countable and Urysohn-closed if each sequence in $X$ u-accumulates to some point $p \in X$.

Proof. Suppose that $X$ is not Urysohn-closed. Then there exists a first countable Urysohn space $Y$ and a homeomorphism $h: X \rightarrow h(X) \subset Y$ such that $h(X)$ is not closed in $Y$. Thus there exists a point $p \in Y-h(X)$ (where $p \in \operatorname{cl}(h(X)))$ and a sequence, $f: N \rightarrow h(X)$, in $h(X)$ converging to $p$. Since 
$h(X)$ is homeomorphic to $X$, the sequence $f u$-accumulates to some point $z \in h(X)$. Therefore $z=p$ according to Theorem 2.5(a), which is a contradiction.

We say that a point $p \in X$ is a $u$-cluster point of $K \subset X$ if for every ordered pair of open sets $(G, H)$ containing $p, \operatorname{cl}(H) \cap(K-\{p\}) \neq \varnothing$. We note that in a Urysohn space $X$, a point $p \in X$ is a $u$-cluster point of $K \subset X$ if and only if for each ordered pair of open sets $(G, H)$ containing $p$, the closure of $H$ contains infinitely many points of $K$.

LEMMA 4.3. In a topological space $X$ the following are equivalent:

(a) Every countably infinite subset of $Y$ has at least one u-cluster point.

(b) Every sequence in $X$ has a u-accumulation point.

THEOREM 4.4. A first countable Urysohn space $X$ is first countable and Urysohn-closed if every countably infinite subset of $Y$ has at least one $u$-cluster point.

Proof. The result follows from Theorem 4.2 and Lemma 4.3.

Theorem 6.3 of $[1$, p. 107] shows that a first countable Urysohn space $X$ is first countable and minimal Urysohn if every countable open Urysohn filterbase on $X$ with a unique accumulation point is convergent. We show (after Lemma 4.5) that a space $X$ is first countable and minimal Urysohn if each sequence in $X$ with a unique $u$-accumulation point is convergent.

Lemma 4.5. If a Urysohn space $X$ has the property that every sequence in $X$ with a unique $u$-accumulation point is convergent, then every sequence in $X$ has a u-accumulation point.

Proof. Suppose $\left(x_{n}\right)$ is a sequence in $X$ with no $u$-accumulation point. Fix $p \in X$ and define a sequence, $\left(z_{n}\right)$, by $z_{n}=p$ if $n$ is odd and $z_{n}=x_{n / 2}$ if $n$ is even. It is clear that $p$ is the unique $u$-accumulation point of $\left(z_{n}\right)$ and that $\left(z_{n}\right)$ does not converge to $p$.

TheOREM 4.6. A first countable Urysohn space $(X, \tau)$ is first countable and minimal Urysohn if every sequence in $X$ with a unique u-accumulation point is convergent.

Proof. Suppose that $h:(X, \tau) \rightarrow(Y, \sigma)$ is a bijective continuous mapping onto a first countable Urysohn space $(Y, \sigma)$. We need to show that $h^{-1}$ is continuous. Let $\left(y_{n}\right)$ be a sequence in $Y$ converging to $y \in Y$. The continuity of $h$ shows that the sequence, $\left(h^{-1}\left(y_{n}\right)\right)$, has the unique $u$-accumulation point $h^{-1}(y)$. By hypothesis, $\left(h^{-1}\left(y_{n}\right)\right)$ converges to $h^{-1}(y)$ showing that $h^{-1}$ is continuous.

\section{REFERENCES}

1. M. P. Berri, J. R. Porter and R. M. Stephenson, Jr., A survey of minimal topological spaces, General Topology and its Relations to Modern Analysis and Algebra, III (Proc. Conf. Kanpur, 1968), Academia, Prague, 1971, pp. 93-114. MR 43 \#3985.

2. J. Dugundji, Topology, Allyn and Bacon, Boston, Mass., 1968. MR 33 \# 1824.

3. C. T. Scarborough, Minimal Urysohn spaces, Pacific J. Math. 27 (1968), 611-617. MR 38 \#6530.

Department of Mathematics, University of Arkansas, Pine Bluff, Arkansas 71601 